\title{
ARTICLE
}

\section{Objectivity as Independence}

\author{
Alexander Reutlinger (i) \\ Munich Center for Mathematical Philosophy, Fakultät für Philosophie, Wissenschaftstheorie und \\ Religionswissenschaft, Ludwig-Maximilians-Universität München (LMU Munich), Ludwigstr. 31, 80539 \\ München, Germany \\ Email: Alexander.Reutlinger@lmu.de
}

(Received 26 May 2020; revised 3 December 2020; accepted 23 January 2021)

\begin{abstract}
Building on Nozick's invariantism about objectivity, I propose to define scientific objectivity in terms of counterfactual independence. I will argue that such a counterfactual independence account is (a) able to overcome the decisive shortcomings of Nozick's original invariantism and (b) applicable to three paradigmatic kinds of scientific objectivity (that is, objectivity as replication, objectivity as robustness, and objectivity as Mertonian universalism).
\end{abstract}

Keywords: Scientific objectivity; invariance; counterfactual dependence; independence; evidence; confirmation; replication; robustness; universalism; ethos of science

\section{Introduction: From invariantism to the counterfactual independence account of scientific objectivity}

In this paper, I will argue for an account of scientific objectivity, according to which objectivity can be defined in terms of counterfactual independence.

This counterfactual independence account is inspired by Nozick's invariantism about objectivity: "an objective fact is one that is invariant under all admissible transformations" (Nozick 2001: 82). To pick one of Nozick's own examples, the shape of a bottle is objective iff the shape remains the same (that is, invariant) if the bottle is rotated or moved in different (actual or possible) ways (Nozick 2001: 78).

However, Nozick's invariantism suffers from at least three shortcomings:

1. Nozick does not provide an explication of the central modal notion of invariance. He merely illustrates the notion of invariance by way of example (such as the bottle example above).

2. Nozick distinguishes between invariant facts (such as the shape of a bottle) and varying facts (for instance, different locations of a bottle). But his account does not restrict which kinds of facts may play these 'roles'. This lack of restriction gives rise to a worry: objectivity is quite cheap. For instance, my political convictions would turn out to be objective because they would remain the same if the 
location of a grain of sand on Mars changes or were to change. This sounds implausible.

3. Nozick's choice of examples suffers from a one-sided diet, because all of his elaborate examples are from physics (Nozick 2001: 77-8, 82-7). Examples from other sciences are sparse (for instance, Nozick 2001: 90-1, 95, 108). Such a one-sided diet is dissatisfying if one is after a general explication of scientific objectivity, as opposed to objectivity in physics only.

These shortcomings motivate three tasks:

- to provide an explication of invariance (explication task),

- to propose a restriction on which kinds of facts can play the role of invariant and varying facts (restriction task), and

- to discuss examples of objectivity that generalize from physics to other sciences (generalizability task).

To address these tasks, I will make an assumption. Following the mainstream in the literature on scientific objectivity (for instance, among many others, Rorty 1980; Daston and Galison 2007), I will assume that scientific objectivity is an epistemic notion. It is a notion characterizing empirical evidence and evidential support relations (i.e. justificatory relations) between a body of evidence and some hypothesis. ${ }^{1}$ I distinguish scientific objectivity from the (non-epistemic) notion of ontological objectivity (that is, mindindependent existence, as referred to in various debates on realism, such as scientific, moral, and mathematical realism). Ontological objectivity is not my topic in this paper, but one advantage of the counterfactual independence account consists in that it points towards a way of understanding this kind of objectivity (see section 5).

Assuming that scientific objectivity is an epistemic notion, I will argue that the counterfactual independence account successfully accomplishes the three above-mentioned tasks when defining scientific objectivity as follows:

Evidence $\mathrm{E}$ is scientifically objective in relation to a given contrast class of scientists and methods if and only if:

1. Independence Condition: $\mathrm{E}$ is counterfactually independent of the scientists, or of the methods they use.

2. Absence Condition: There is no evidence of defeaters.

In this paper, I will explicate and motivate the Independence Condition and the Absence Condition.

More precisely, the plan of the paper is as follows: in section 2, I will explicate how the Independence Condition of the counterfactual independence account solves the explication task and the restriction task. In section 3, I will address the generalizability task by arguing that the Independence Condition is applicable to three paradigmatic examples of objectivity that can be found in various sciences, not only in physics. In section 4, I will articulate and motivate the Absence Condition. Section 5 provides a conclusion and an outlook on three advantages of the counterfactual independence account.

My main goal in this paper is fairly modest: to defend the counterfactual independence account as a theory of scientific objectivity that meets the three tasks introduced

\footnotetext{
${ }^{1} \mathrm{My}$ focus is on an epistemic notion of objectivity in scientific contexts. This focus does not exclude that an epistemic notion of objectivity is also used in non-scientific contexts, such as juridical contexts.
} 
above. It is not my ambition to assess the merit of the counterfactual independence account relative to alternative accounts of scientific objectivity in any great detail. For brevity's sake, I will leave this ambitious project to another paper.

However, I would like to highlight a feature of the counterfactual independence account in order to situate it in the literature on objectivity. The counterfactual independence account provides a unifying account of objectivity in science, since it captures different kinds of objectivity on the basis of a single general, precise and informative explication of what makes these different kinds of objectivity objective, or so I argue. This unificationist spirit aims at an alternative to other approaches to scientific objectivity, according to which there is no general, precise and informative story to tell (such as, among others, Douglas 2004; Hacking 2015).

\section{Explicating the Independence Condition}

In this section, I will argue that the Independence Condition allows me to meet the explication task and the restriction task.

Regarding the explication task, I propose to explicate Nozick's undefined modal notion of invariance as counterfactual independence. It is useful to introduce the notion of counterfactual dependence first in order to define counterfactual independence in a second step.

Fact A counterfactually depends on fact B iff the following counterfactual conditionals are true: (1) if B were the case, then A would be the case, and (2) if B were not the case, then A would not be the case.

This notion of dependence is familiar from counterfactual theories of causation and explanation (for instance, Lewis 1973; Woodward 2003). ${ }^{2}$ We are now in a position to define what it means that $\mathrm{A}$ does not counterfactually depend on $\mathrm{B}$, or equivalently, and to use my preferred label, what it means that A is counterfactually independent of B:

A is counterfactually independent of B iff the following counterfactual conditionals are true: (1) if B were the case, then A would be the case, and (2) if B were not the case, then A would (still) be the case.

Furthermore, I take it to be useful to attribute the following connected features to counterfactual independence:

- Independence is relational. The sentence " $\mathrm{A}$ is independent" does not make sense or is at least incomplete. Only " $\mathrm{A}$ is independent of $\mathrm{B}$ (or, in relation to $\mathrm{B}$ )" is complete and meaningful. Hence, A might be independent of $\mathrm{B}$ but not of some other fact $\mathrm{C}$.

- Independence requires a contrast class. Suppose A is independent of B. Asserting such independencies (explicitly or implicitly) relies on a relevant contrast class whose elements specify the possible alternatives to B, or possible variations of $\mathrm{B}$ (van Fraassen 1980; similarly, Lipton 2004). Thereby, the contrast class indicates which antecedents matter for the counterfactual conditionals if we want to assert that $A$ is independent of $B$. In the simplest case, the contrast class of $B$ is $\{$ not- $B$. But, as I will illustrate in section 3, in realistic examples of scientific objectivity the relevant contrast class tends to have more members.

\footnotetext{
${ }^{2}$ I will remain neutral between different semantics for counterfactual conditionals.
} 
- Independence is comparative. Contrast classes may differ, for instance, in how many (and which) elements they have (Nozick 2001: 87, 99). If so, then some fact $A$ might be independent of fact $B_{1}$ given the small contrast class $\left\{B_{2}\right\}$. Some other fact $A^{*}$ might display independence of $B_{1}$ given a contrast class with more elements, such as the class $\left\{B_{2} ; B_{3}\right\}$ that has $\left\{B_{2}\right\}$ as a proper subset. In such a case, we can compare $A^{*}$ s and $A^{\prime}$ s independencies directly and state that $\mathrm{A}^{\star}$ is independent of more varying facts than $\mathrm{A}^{3}$

If objectivity is defined in terms of independence, as I propose, then objectivity inherits these three features.

Although the notion of counterfactual independence (and its synonyms such as stability and resilience) has not been explicitly used to define scientific objectivity, it is at the heart of various attempts to capture other central concepts in the philosophy of science, such as laws of nature (see, for instance, Skyrms 1980; Lange 2009; see also, at least implicitly, Nozick 2001: 85-6).

Let me now turn to the restriction task. Assuming that scientific objectivity is an epistemic notion (see section 1), I propose the following restriction on the kinds of facts that are counterfactually independent of one another: the invariant facts are facts concerning empirical evidence (for some hypothesis), whereas the (counterfactually) varying facts concern (a) the different (possible) scientists (or groups of scientists) who do the research to obtain the evidence, and/or (b) the different methods scientists use (illustrations will follow in section 3).

Merging my answers to the explication task and the restriction task, we arrive at the Independence Condition, according to which evidence E is counterfactually independent of the scientists, or of the methods they use. The Independence Condition provides a more restrictive and more precise explication of the key idea of Nozick's invariantism: an objective fact is no longer understood as some arbitrary fact "that is invariant under all admissible transformations" (Nozick 2001: 82); instead, an objective fact is defined more restrictively as a fact about empirical evidence that is counterfactually independent of scientists that produce it or the methods that they use.

\section{Applying the Independence Condition to three kinds of objectivity in science}

Now, I will take up the generalization task by applying the Independence Condition to three kinds of objectivity that occur frequently in the (experimental) sciences, not just in physics. I will argue that the Independence Condition captures at least three paradigmatic kinds of objectivity: objectivity as replication, objectivity as robustness, and objectivity as Mertonian universalism. ${ }^{4}$

The first kind of objectivity - objectivity as replication - consists in the successful replication of experimental results that serve as evidence. That is, a scientist $\mathrm{S}_{2}$ (or, more realistically, a group of scientists) is able to replicate the experimental result $\mathrm{E}$ of another scientist $S_{1}$ (or another group of scientists) by using the same method $M$

\footnotetext{
${ }^{3}$ Another scenario is possible: $\mathrm{A}^{*}$ is independent of more varying facts than $\mathrm{A}$, but the contrast class of $\mathrm{A}$ is not a proper subset of the contrast class of $A^{\star}$. This is a less interesting scenario, because it does not allow a direct comparison of $A^{\star \prime} s$ and $A^{\prime}$ 's independencies.

${ }^{4}$ I will present these kinds of objectivity in a 'stylized' way: that is, I will work with an abstract description of each kind. I will not provide detailed case studies for each kind.
} 
that $\mathrm{S}_{1}$ applied. ${ }^{5}$ That is, in replication cases, the varying facts concern different scientists, not the methods used (because $S_{1}$ and $S_{2}$ use the same method $M$ ).

The counterfactual independence account captures objectivity as replication in the following manner appealing to the Independence Condition: evidence E, obtained by using experimental method $M$, is objective relative to the contrast class \{scientist $S_{1}$; scientist $S_{2}$ \} iff obtaining $E$ is independent of whether $S_{1}$ or $S_{2}$ applies method $M-$ that is, the following two counterfactual conditionals have to be true: (1) if $S_{1}$ used experimental method $M$, then $E$ would be the experimental result, and (2) if $S_{2}$ used $M$, then $E$ would also be the experimental result.

Objectivity as robustness, the second kind of objectivity, consists in establishing that an experimental result, evidence $\mathrm{E}$, is robust. That is, different (groups of) scientists are able to achieve the same result in their labs by using different methods. ${ }^{6}$ That is, in case of robustness, the varying facts mainly concern the methods being used; the varying facts might also concern the scientists (or a group of scientists), but it is possible that a single scientist (or a group of scientists) arrives at robust research results by applying different methods. I will apply the counterfactual independence account to a type of robustness where the varying facts include methods and scientists.

According to the Independence Condition, we can capture objectivity as robustness as follows: experimental result $\mathrm{E}$ is objective in relation to the contrast class \{scientist $S_{1}$; scientist $S_{2}$; method $M_{1}$; method $M_{2}$ \} iff (1) if $S_{1}$ were to use $M_{1}$, then $E$ would be the result, and (2) if $S_{2}$ were to use $M_{2}$, then $E$ would also be the result (or some equivalent piece of evidence $E^{\prime}$ that can be mapped onto the result $E$ of applying $M_{1}$ ).

Objectivity as Mertonian universalism - the third kind - is the requirement that certain features of scientists should not matter when they assess the evidence. For instance, a scientist's nationality or social class should not matter for this activity. Merton famously expresses this point as the rule of "universalism" that is part of the "ethos of science":

The acceptance or rejection of claims entering the lists of science is not to depend on the personal or social attributes of their protagonist; his race, nationality, religion, class, and personal qualities are as such irrelevant. (Merton 1942 [1973]: 270)

Different expressions of universalism can also be found in other prominent places in the literature. ${ }^{7}$

Is the counterfactual independence account able to capture universalism? In the context of this paper, universalism might be best understood as an additional constraint on other kinds of objectivity, such as objectivity as replication and objectivity as robustness. According to the counterfactual independence account, objectivity as universalism consists in adding further elements to the contrast class. This strategy for describing universalism applies to both objectivity as replication and objectivity as robustness.

Suppose that two scientists $S_{1}$ and $S_{2}$ differ in certain features $F_{1}$ and $F_{2}$, in their "personal or social attributes" (Merton 1942 [1973]). For instance, two scientists might differ with respect to their nationality, social class, gender, the sponsors funding their research (for instance, public funding versus industry funding), and so on.

In the case of objectivity as replication, Mertonian universalism simply enlarges the relevant contrast class; it becomes \{scientist $S_{1}$; scientist $S_{2}$; feature $F_{1}$; feature $\left.F_{2}\right\}$, and

\footnotetext{
${ }^{5}$ See Carnap (1932: 446), Popper (1934: 18-19), Rorty (1980: 333-42), Shapin and Schaffer (1985: 5960); and more recently Douglas (2004: 462) and Lloyd and Schweizer (2014: 2069-70).

${ }^{6}$ See Carnap (1932: 446-7); in the current literature, see Douglas (2004: 458) and Wimsatt (2007: Ch. 4).

${ }^{7}$ For instance, Weber (1949: 58), Longino (1990: 76-81), Daston (1992: 599), Daston and Galison (1992: 98; 2007: 121); see also Nozick (2001: 96).
} 
the resulting conditionals are (1) if $S_{1}$ with feature $F_{1}$ used experimental method $M$, then $E$ would be the experimental result, and (2) if $S_{2}$ with feature $F_{2}$ used experimental method $\mathrm{M}$, then $\mathrm{E}$ would also be the experimental result.

In the case of objectivity as robustness, the enlarged 'universalist' contrast class is \{scientist $\mathrm{S}_{1}$; scientist $\mathrm{S}_{2}$; feature $\mathrm{F}_{1}$; feature $F_{2}$; method $\mathrm{M}_{1}$; method $\mathrm{M}_{2}$, while the relevant conditionals are: (1) if $S_{1}$ with feature $F_{1}$ were to use $M_{1}$, then E would be the result, and (2) if $S_{2}$ with feature $F_{2}$ were to use $M_{2}$, then $E$ would also be the result.

I take all three kinds of objectivity to be representative, as they are perceived as being widespread and paradigmatic in the literature on objectivity in philosophy and history of science. Of course, there might be further kinds of objectivity in science, as indicated in the recent literature in philosophy and history of science: for instance, "structural objectivity" (Daston and Galison 2007; Padovani et al. 2015), objectivity as different kinds of "witnessing" in science (Shapin and Schaffer 1985: 55-65), and objectivity as expert agreement in the context of assessment reports (such as the IPCC report, see Oppenheimer et al. 2019). For this reason, I take it to be a fruitful task for future research to explore whether the counterfactual independence account can also be applied to further kinds of objectivity.

In sum, I have argued for the claim that the Independence Condition applies to three pervasive kinds of scientific objectivity. This result helps to address the generalizability task, because all three kinds of objectivity can be found in various (experimental) sciences, not merely in physics. Moreover, applying the Independence Condition to the three kinds of objectivity illustrates that independence is relational (because each kind of objectivity identifies certain facts relative to which the evidence is objective), that stating independencies requires a contrast class (because a relevant contrast class has to be chosen) and that independence has a comparative character (because the kinds of objectivity suggest contrast classes with a different number of members).

\section{Motivating the Absence Condition}

The counterfactual independence account, as presented in section 1, also rests on a second necessary condition: the Absence Condition. According to this condition, we need to be aware of the fact that claims about independencies (required by the Independence Condition) are defeasible and, moreover, that objectivity requires that there be no evidence of defeaters. Consider two examples of defeaters for illustration. For simplicity's sake, I will focus on objectivity as replication in these examples.

First, suppose that scientist $S_{1}$ first learns that a fellow scientist $S_{2}$ has successfully replicated her experimental study. She rightly takes this new information to be an indication of scientific objectivity. Then, however, $S_{1}$ also learns that $S_{2}$ has been working with a method that is known to be highly unreliable. Hence, the 'replication' was merely a matter of luck. This second piece of information plays the role of a defeater, as it makes $S_{1}$ considerably less confident that the (seeming) replication establishes the objectivity of the empirical results of her own study (for a historical case study, see Daston and Galison 2007: 11-16, 154-61; Reutlinger 2020: section 3.1).

Second, imagine that $S_{1}$ learns that $S_{2}$ failed to replicate the experimental result $E$ of her original empirical study. $S_{1}$ counts this information as indicating a failure of objectivity. But imagine that $S_{1}$ then obtains evidence that $S_{2}$ is funded by a company (whose non-epistemic interests are in conflict with the result $\mathrm{E}$ of her original study) and that $S_{2}$ has omitted a relevant part of the data (for real cases of biased research, see Oreskes and Conway 2010; Reutlinger 2020: section 3.2). For $S_{1}$, the second piece of information is defeating evidence: now, $S_{1}$ has a good reason not to treat the result of $S_{2}$ 's study as undermining the objectivity of her original study. Whether her original study did 
indeed produce objective evidence depends on whether it will be successfully replicated in the future.

Both examples suggest that claims about counterfactual independence are defeasible. For this reason, a proponent of the counterfactual independence account should demand that there be no evidence of defeaters. This is the second necessary condition - the Absence Condition - I impose on scientific objectivity. ${ }^{8}$

\section{Conclusion and outlook}

I have proposed the counterfactual independence account and I have argued that this account is able to overcome the shortcomings of Nozick's invariantism. For this reason alone, I believe it is an account of scientific objectivity that deserves further discussion.

Another attractive feature of the counterfactual independence account consists in the fact that it provides a unificationist approach to objectivity (as outlined in section 1): that is, it is designed to provide an alternative to other approaches in the historical and philosophical literature, according to which there is no general, precise and informative account of scientific objectivity. To advance the unificationist project, it will be fruitful to apply the counterfactual independence account to further kinds of objectivity (see section 3 for examples). ${ }^{9}$

Finally, let me stress three advantageous and fruitful consequences of the counterfactual independence account for future research.

First, the account explains why scientist strive for objectivity: valuing objectivity is simply a plea for more evidence, for more evidence from different sources (from different scientists and/or methods). Hence, if scientists care about empirical evidence, it is not surprising that they value objectivity. It might be fruitful to elaborate this idea on the basis of extant accounts of empirical confirmation (such as Bayesian confirmation theory, frequentist hypothesis testing and likelihoodism).

Second, the counterfactual independence account can be adopted by both scientific realists and scientific anti-realists, because objectivity is characterized by observable facts (facts about evidence, scientists, methods, and the absence or presence of defeaters). Hence, the account I propose is neutral with respect to the persistent disagreement about scientific realism. I take this to be an advantage of defining objectivity in terms of the counterfactual independence account.

Third, although I have focused on scientific objectivity as an epistemic notion, the counterfactual independence account also opens up a novel way to understand ontological objectivity. Suppose that some fact A is ontologically objective - that is, A exists mind-independently. Proponents of the counterfactual independence account might adopt the following approach to ontological objectivity: A is ontologically objective relative to the contrast class \{there are mental states concerned with $\mathrm{A}$; there are no mental states concerned with A\} iff (1) if there were mental states concerned with A, then A would obtain, and (2) if there were no mental states concerned with $A$, then $A$ would still obtain. Surely, this is not the final word on ontological objectivity but it

\footnotetext{
${ }^{8}$ Whether the Absence Condition is indeed satisfied has to be determined on the level of analyzing concrete case studies exemplifying the 'stylized' kinds of objectivity discussed in section 3. It is, however, not my goal to provide such an analysis in this paper (I have done this elsewhere; see Reutlinger 2020).

9Objectivity as "virtual witnessing" (Shapin and Schaffer 1985: 60-5) and as expert agreement in the context of assessment reports (Oreskes and Conway 2010; Oppenheimer et al. 2019) are particularly challenging kinds of objectivity. This is the case because the methods scientists use do not consist in conducting experiments but instead in assessing and understanding the evidence as it is presented in scientific publications. To explore whether the counterfactual independence account applies to such cases is a project worth undertaking.
} 
is a direction worth exploring - a direction that might spark a debate among philosophers of science and metaphysicians. ${ }^{10}$

\section{References}

Carnap R. (1932). 'Die Physikalische Sprache als Universalsprache der Wissenschaft.' Erkenntnis 2, 432-65.

Daston L. (1992). 'Objectivity and the Escape from Perspective.' Social Studies of Science 22, 597-618.

Daston L. and Galison P. (1992). 'The Image of Objectivity.' Representations 40, 81-128.

Daston L. and Galison P. (2007). Objectivity. New York, NY: Zone Books.

Douglas H. (2004). 'The Irreducible Complexity of Objectivity.' Synthese 138, 453-73.

Hacking I. (2015). 'Let's Not Talk About Objectivity.' In F. Padovani, A. Richardson and J. Y. Tsou (eds), Objectivity in Science: New Perspectives from Science and Technology Studies, pp. 19-33. New York, NY: Springer.

Lange M. (2009). Laws and Lawmakers. Oxford: Oxford University Press.

Lewis D. (1973). 'Causation.' In Philosophical Papers II, pp. 159-72. Oxford: Oxford University Press.

Lipton P. (2004). Inference to the Best Explanation. 2nd Edition. London: Routledge.

Lloyd E. and Schweizer V. (2014). 'Objectivity and a Comparison of Methodological Scenario Approaches for Climate Change Research.' Synthese 191, 2049-88.

Longino H. (1990). Science as Social Knowledge. Princeton, NJ: Princeton University Press.

Merton R.K. (1942 [1973]). 'The Normative Structure of Science.' In The Sociology of Science: Theoretical and Empirical Investigations, pp. 267-78, Chicago, IL: The University of Chicago Press.

Nozick R. (2001). Invariances: The Structure of the Objective World. Cambridge, MA: Harvard University Press.

Oppenheimer M., Oreskes N., Jamieson D., Brysse K., O’Reilly J., Shindell M. and Wazeck M. (2019). Discerning Expert: The Practices of Scientific Assessment for Environmental Policy. Chicago, IL: The University of Chicago Press.

Oreskes N. and Conway E. (2010). Merchants of Doubt: How a Handful of Scientists Obscured the Truth on Issues from Tobacco Smoke to Global Warming. New York, NY: Bloomsbury Press.

Padovani F., Richardson A. and Tsou J. (eds) (2015). Objectivity in Science: New Perspectives from Science and Technology Studies. Cham: Springer.

Popper K. (1934). Logik der Forschung. Tübingen: Mohr Siebeck.

Reutlinger A. (2020). 'What Is Epistemically Wrong With Research Affected By Sponsorship Bias? The Evidential Account.' European Journal for Philosophy of Science 10. doi.org/10.1007/s13194-020-00280-2.

Rorty R. (1980). Philosophy and the Mirror of Nature. Oxford: Basil Blackwell.

Shapin S. and Schaffer S. (1985). Leviathan and the Air-Pump: Hobbes, Boyle, and the Experimental Life. Princeton, NJ: Princeton University Press.

Skyrms B. (1980). Causal Necessity. New Haven, CT: Yale University Press.

Van Fraassen B. (1980). The Scientific Image. Oxford: Oxford University Press.

Weber M. (1949). On the Methodology of the Social Sciences. Glencoe/IL: The Free Press.

Wimsatt W. (2007). Re-Engineering Philosophy for Limited Beings. Cambridge, MA: Harvard University Press.

Woodward J. (2003). Making Things Happen: A Theory of Causal Explanation. New York, NY: Oxford University Press.

Alexander Reutlinger is Akademischer Rat (a.L.) at the Munich Center for Mathematical Philosophy (LMU Munich). His research is concerned with topics in philosophy of science and neighboring areas of epistemology, metaphysics, and political philosophy.

\footnotetext{
${ }^{10}$ I would like to thank Maria Kronfeldner for very constructive and repeated feedback on different drafts. The comments of the editor and referees helped a lot to improve the quality of the manuscript. Moreover, I did benefit from discussions with my colleagues at the Munich Center for Mathematical Philosophy, the participants of a seminar on objectivity I co-taught with Kärin Nickelsen at LMU Munich, and kind audiences in Budapest, Cologne, Hannover, Munich, and Vienna. Many thanks to Andreas Hüttemann for drawing my attention to Nozick's book and for a conversation about it.
}

Cite this article: Reutlinger A (2021). Objectivity as Independence. Episteme 1-8. https://doi.org/10.1017/ epi.2021.5 